\title{
VLBI observations of weak sources using fast frequency switching
}

\author{
E. Middelberg ${ }^{1, \star}$, A. L. Roy ${ }^{1,2}$, R. C. Walker ${ }^{3}$, and H. Falcke ${ }^{4}$ \\ 1 Max-Planck-Institut für Radioastronomie, Auf dem Hügel 69, 53121 Bonn, Germany \\ e-mail: enno.middelberg@csiro.au; aroy@mpifr-bonn.mpg.de \\ 2 Geodätisches Institut der Universität Bonn, Nussallee 17, 53115 Bonn, Germany \\ 3 National Radio Astronomy Observatory, PO Box 0, Socorro, NM, 87801, USA \\ e-mail: cwalker@aoc.nrao.edu \\ 4 ASTRON, PO Box 2, 7990 AA Dwingeloo, The Netherlands \\ e-mail: falcke@astron.nl
}

Received 28 September 2004 / Accepted 17 December 2004

\begin{abstract}
We have developed a new phase referencing technique for high frequency VLBI observations. In conventional phase referencing, one interleaves short scans on a nearby phase calibrator between the target source observations. In fast frequency switching described here, one observes the target source continuously while switching rapidly between the target frequency and a lower reference frequency. We demonstrate that the technique allows phase calibration almost reaching the thermal noise limit and present the first detection of the AGN in the FR I radio galaxy NGC 4261 at $86 \mathrm{GHz}$. Although point-like, this is the weakest source ever detected with VLBI at this frequency.
\end{abstract}

Key words. techniques: interferometric - techniques: high angular resolution - methods: observational - galaxies: active galaxies: jets - galaxies: individual: NGC 4261

\section{Introduction}

The regions where jets from active galactic nuclei (AGN) are launched and collimated are difficult to observe with VLBI because they lie very close to the black hole and most bright objects are very distant. Thus, there are still very few observational constraints on jet formation. Only in the closest AGN can the highest resolution observations resolve several tens of Schwarzschild radii $\left(R_{\mathrm{S}}\right)$, comparable to the scale of $10 R_{\mathrm{S}}$ to $1000 R_{\mathrm{s}}$ where jet formation is expected to take place (e.g., Koide et al. 2000; Appl \& Camenzind 1993).

One of the best targets for resolving details in the jet is NGC 4261 (3C 270). It is an elliptical low-luminosity FR I radio galaxy hosting a $4.9 \times 10^{8} M_{\odot}$ black hole (Ferrarese et al. 1996) which powers a double-sided radio jet (Jones \& Wehrle 1997; Jones et al. 2000, 2001). Given the distance to NGC 4261 of $28.2 \mathrm{Mpc}\left(H_{0}=75 \mathrm{~km} \mathrm{~s}^{-1} \mathrm{Mpc}^{-1}\right)$ and its black hole mass, $86 \mathrm{GHz}$ VLBI observations are expected to resolve $200 R_{\mathrm{s}}$. This measurement would be, after diameter measurements of Sgr A* $\left(24 R_{\mathrm{s}}\right.$, Bower et al. 2004, $17 R_{\mathrm{s}}$, Krichbaum et al. 1998) and M 87 (32 $R_{\mathrm{s}}$, Junor et al. 1999, 46 $R_{\mathrm{s}}$, Ly et al. 2004) the third-highest resolution image ever achieved in terms of Schwarzschild radii. Furthermore, the high inclination of the

^ Present address: Australia Telescope National Facility, PO Box 76, Epping NSW 1710, Australia. jets makes NGC 4261 a good candidate to look for a coreshift with frequency. Core-shift measurements are of considerable astrophysical interest. They can help to discriminate between the conically-expanding jet model (Blandford \& Königl 1979) and advection-dominated accretion flows (ADAFs, e.g., Narayan et al. 1998) in low-luminosity AGN, to find the true location of the AGN, to determine the jet magnetic field strength and to test for potential foreground absorbers (Lobanov 1998). Although NGC 4261 therefore is an interesting source to study the jet formation process, it is unfortunately not possible to directly observe NGC 4261 with $86 \mathrm{GHz}$ VLBI, because it is too weak. This paper demonstrates a new calibration method that makes such an observation possible.

High frequency observations involve a number of serious problems: the sources are usually weak because the emissivity of optically thin synchrotron sources drops as $v^{-0.7}$, the aperture efficiencies of most of the radio telescopes drop to $15 \%$ or less because their surface accuracies were specified for $\mathrm{cm}$-wavelength operation, the receiver performances become disproportionately worse because of higher amplifier noise, the atmospheric contribution to the system temperatures increases towards higher frequency, and the atmospheric coherence that limits the integration time decreases as $1 / v$.

However, the integration times could be prolonged if the atmospheric phase fluctuations could be calibrated. This can be 
done using self-calibration, or if the source is too weak, then using phase referencing (e.g., Shapiro et al. 1979; Marcaide \& Shapiro 1984; Alef 1988). Both are established calibration techniques and phase referencing can be used to lower detection thresholds to the sub-mJy level and can accurately determine source positions. In phase referencing, a strong calibrator source is observed frequently (every few minutes, depending on observing frequency) to calibrate the visibility phases of the target source integrations, i.e., the telescopes cycle between the target source and the phase calibrator source. In millimetre VLBI the technique is not commonly used because of the need for a suitable, strong phase calibrator in the vicinity of the target source and from a combination of short atmospheric coherence time and relatively long telescope slewing times. However, a successful proof of concept exists for $86 \mathrm{GHz}$ VLBI observations (Porcas \& Rioja 2002).

In this paper, we describe a novel way of calibrating visibility phases to overcome these limitations, using interleaved observations at a low and a high frequency.

\section{Principle of phase correction}

\subsection{Self-calibration}

The phase of the complex visibility function which an interferometer measures is altered by instrumental drifts, antenna and source position errors, and path length changes in the earth's atmosphere. These errors can be accounted for using self-calibration, which is similar to adaptive optics in optical astronomy. Starting with a point source model in the field centre, and refining the model iteratively, antenna-based correction phases are derived that make the visibility phases compliant with the model. Similar to adaptive optics, where a sufficiently bright guide star is required to detect the shape of the wavefront, self-calibration requires a minimum signal-to-noise ratio (SNR) on each baseline. Visibilities with an SNR of less than about five within the atmospheric coherence time are commonly regarded as non-detections.

\subsection{Scaling and interpolation}

One can self-calibrate the visibility phases at one frequency and use the solutions to calibrate visibilities at another frequency after multiplying the phases by the frequency ratio, $r$. This works since the main source of phase noise in VLBI observations at frequencies higher than about $5 \mathrm{GHz}$ is turbulence in the troposphere causing refractive inhomogeneities which are non-dispersive. One requires that the lag between the two measurements does not exceed half the atmospheric coherence time (Fig. 1). Instead of using a phase-referencing calibrator source, the source is phase-referenced to itself at a lower frequency.

This is possible with the Very Long Baseline Array ${ }^{1}$ (VLBA, Napier et al. 1994) because frequency changes need only a few seconds, in which the subreflector is moved from one feed horn to the other, and because the local oscillator

\footnotetext{
${ }^{1}$ The VLBA is an instrument of the National Radio Astronomy Observatory, a facility of the National Science Foundation, operated under cooperative agreement by Associated Universities, Inc.
}

14.375 vs. 43.125 GHz Visibility Phases
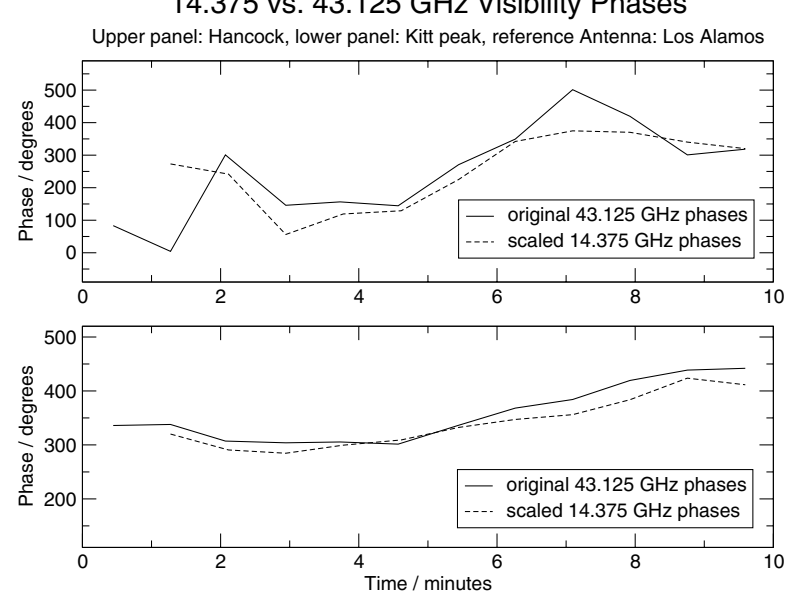

Fig. 1. Demonstration of the scalability of phase solutions. $43 \mathrm{GHz}$ fringe-fitted phase solutions (solid lines) on 3C 273 compared to $15 \mathrm{GHz}$ fringe-fitted phase solutions multiplied by the frequency ratio (dashed lines) from the VLBA antennas at Hancock (upper panel) and Kitt Peak (lower panel) to the reference VLBA antenna at Los Alamos. The phases follow each other very well.

phase returns to its original setting after frequency switching. After multiplying the phase solutions by the frequency ratio and applying them to the target-frequency phases, there remains a constant phase offset, $\Delta \Phi$, between the signal paths at the two frequencies, which must be calibrated. This can be monitored with frequent observations of achromatic, strong calibrators, and must be subtracted from the high-frequency visibility phase.

The observed visibility phases using this calibration scheme can be described as

$$
\begin{aligned}
\phi_{\mathrm{r}}^{\mathrm{obs}}\left(t_{1}\right)=\phi_{\mathrm{r}}^{\mathrm{tru}}\left(t_{1}\right)+\phi_{\mathrm{r}}^{\mathrm{ins}}\left(t_{1}\right) & +\phi_{\mathrm{r}}^{\mathrm{pos}}\left(t_{1}\right)+\phi_{\mathrm{r}}^{\mathrm{ant}}\left(t_{1}\right) \\
& +\phi_{\mathrm{r}}^{\mathrm{tro}}\left(t_{1}\right)+\phi_{\mathrm{r}}^{\mathrm{ion}}\left(t_{1}\right) \\
\phi_{\mathrm{t}}^{\mathrm{obs}}\left(t_{2}\right)=\phi_{\mathrm{t}}^{\mathrm{tru}}\left(t_{2}\right)+\phi_{\mathrm{t}}^{\mathrm{ins}}\left(t_{2}\right) & +\phi_{\mathrm{t}}^{\mathrm{pos}}\left(t_{2}\right)+\phi_{\mathrm{t}}^{\mathrm{ant}}\left(t_{2}\right) \\
& +\phi_{\mathrm{t}}^{\mathrm{tro}}\left(t_{2}\right)+\phi_{\mathrm{t}}^{\mathrm{ion}}\left(t_{2}\right) \\
\phi_{\mathrm{r}}^{\mathrm{obs}}\left(t_{3}\right)=\phi_{\mathrm{r}}^{\mathrm{tru}}\left(t_{3}\right)+\phi_{\mathrm{r}}^{\mathrm{ins}}\left(t_{3}\right) & +\phi_{\mathrm{r}}^{\mathrm{pos}}\left(t_{3}\right)+\phi_{\mathrm{r}}^{\text {ant }}\left(t_{3}\right) \\
& +\phi_{\mathrm{r}}^{\mathrm{tro}}\left(t_{3}\right)+\phi_{\mathrm{r}}^{\text {ion }}\left(t_{3}\right)
\end{aligned}
$$

Here, $\phi^{\text {tru }}$ indicates true visibility phases of the source, $\phi^{\text {ins }}$ is the residual instrumental phase error, $\phi^{\text {pos }}$ and $\phi^{\text {ant }}$ are geometric errors arising from source and antenna position errors, and $\phi^{\text {tro }}$ and $\phi^{\text {ion }}$ are tropospheric and ionospheric phase noise contributions. The subscript indices $\mathrm{r}$ and $\mathrm{t}$ indicate quantities that belong to the reference frequency, $v_{\mathrm{r}}$, and the target frequency, $v_{\mathrm{t}}$, respectively.

Self-calibration at the reference frequency is used to obtain a source model and hence to obtain $\phi_{\mathrm{r}}^{\mathrm{tru}}\left(t_{1}\right)$ and $\phi_{\mathrm{r}}^{\mathrm{tru}}\left(t_{3}\right)$. Self-calibration of the visibilities at the reference frequency after subtracting this model yields $\phi_{\mathrm{r}}^{\text {cor }}$, the sum of $\phi_{\mathrm{r}}^{\text {ins }}, \phi_{\mathrm{r}}^{\text {pos }}$, $\phi_{\mathrm{r}}^{\text {ant }}, \phi_{\mathrm{r}}^{\text {tro }}$ and $\phi_{\mathrm{r}}^{\text {ion }}$. This sum is linearly interpolated to the times where the target frequency was observed, and is scaled by the frequency ratio, $r$ :

$r \tilde{\phi}_{\mathrm{r}}^{\text {cor }}=r\left(\tilde{\phi}_{\mathrm{r}}^{\text {ins }}+\tilde{\phi}_{\mathrm{r}}^{\text {pos }}+\tilde{\phi}_{\mathrm{r}}^{\text {ant }}+\tilde{\phi}_{\mathrm{r}}^{\text {tro }}+\tilde{\phi}_{\mathrm{r}}^{\text {ion }}\right)$

where a tilde denotes quantities linearly interpolated to time $t_{2}$. The difference between the target frequency visibility phase 
and the interpolated and scaled reference frequency correction phase at time $t_{2}$ is then

$$
\begin{aligned}
\phi_{\mathrm{t}}^{\mathrm{obs}}-r \tilde{\phi}_{\mathrm{r}}^{\mathrm{cor}}= & \phi_{\mathrm{t}}^{\mathrm{tru}}+\left(\phi_{\mathrm{t}}^{\mathrm{ins}}-r \tilde{\phi}_{\mathrm{r}}^{\mathrm{ins}}\right)+\left(\phi_{\mathrm{t}}^{\mathrm{pos}}-r \tilde{\phi}_{\mathrm{r}}^{\mathrm{pos}}\right) \\
& +\left(\phi_{\mathrm{t}}^{\mathrm{ant}}-r \tilde{\phi}_{\mathrm{r}}^{\mathrm{ant}}\right)+\left(\phi_{\mathrm{t}}^{\mathrm{tro}}-r \tilde{\phi}_{\mathrm{r}}^{\mathrm{tro}}\right) \\
& +\left(\phi_{\mathrm{t}}^{\mathrm{ion}}-r \tilde{\phi}_{\mathrm{r}}^{\mathrm{ion}}\right) .
\end{aligned}
$$

The instrumental phase offset, $\Delta \Phi=\left(\phi_{\mathrm{t}}^{\text {ins }}-r \tilde{\phi}_{\mathrm{r}}^{\text {ins }}\right)$, is constant with time and can be determined from calibrator observations, and so it is known and can be removed. The antenna position error, like all geometric errors, scales with frequency and hence $\left(\phi_{\mathrm{t}}^{\mathrm{ant}}-r \tilde{\phi}_{\mathrm{r}}^{\mathrm{ant}}\right)=0$. A frequency-dependent core shift, however, as predicted by conical jet models (e.g., Lobanov 1998), modulates $\left(\phi_{\mathrm{t}}^{\text {pos }}-r \tilde{\phi}_{\mathrm{r}}^{\text {pos }}\right)$ on each baseline with a sinusoid. The period of the sinusoid is $23^{\mathrm{h}} 56^{\mathrm{m}}$, its amplitude depends on the magnitude of the shift and its phase depends on the direction of the shift relative to the baseline direction. It is zero only in the absence of a core shift, and therefore provides useful structural information. The tropospheric phase errors also scale with frequency, and hence $\left(\phi_{\mathrm{t}}^{\text {tro }}-r \tilde{\phi}_{\mathrm{r}}^{\text {tro }}\right)=0$. The ionospheric phase errors do not scale linearly with frequency and so cannot be removed using this technique, requiring that they be measured separately. We assume for the moment that this has been done, but see the next section for the impact of ionospheric effects on the data presented here. The remaining terms describe the difference between the target frequency phase and the scaled and interpolated reference frequency phase corrections as the target frequency visibility phase plus the position offset:

$\phi_{\mathrm{t}}^{\mathrm{obs}}-r \tilde{\phi}_{\mathrm{r}}^{\mathrm{cor}}=\phi_{\mathrm{t}}^{\mathrm{tru}}+\left(\phi_{\mathrm{t}}^{\mathrm{pos}}-r \tilde{\phi}_{\mathrm{r}}^{\mathrm{pos}}\right)$.

Thus, the true high-frequency visibilities are phase-referenced to the source's low-frequency visibilities, and so the technique can prolong coherence and can measure the position shift of cores in AGN with frequency.

\section{3. lonospheric path length changes}

For illustration, we assumed in the previous section that the ionospheric effects were calibrated. Ionospheric path length changes can probably be determined using interspersed, wide-band scans at a low frequency, e.g., in the $1.4 \mathrm{GHz}$ band, where the effect is strong. We had not appreciated before observing that the ionosphere would still be significant at $15 \mathrm{GHz}$ and had not planned $1.4 \mathrm{GHz}$ scans to measure ionosphere. Hence, the high-frequency visibilities from the observations presented here contained unmodelled ionospheric path length changes which limited the coherence to half an hour in the worst case. To remove the remaining long-term phase drifts and remaining phase offsets required one extra step of self-calibration. This step of self-calibration loses source position information, which prevented a core-shift measurement from being made. Still the extension from the $30 \mathrm{~s}$ atmospheric coherence time to a coherent integration time of $30 \mathrm{~min}$ yielded a large sensitivity improvement and allows weaker sources to be detected.

A similar observing strategy has been developed by Kassim et al. (1993) for the VLA, who used scaled phase solutions from $330 \mathrm{MHz}$ to calibrate simultaneously observed $74 \mathrm{MHz}$ data. In this case, the dominant source of phase errors was ionospheric path length changes. Using their new calibration technique, they were able to increase the coherent integration time from $<1 \mathrm{~min}$ to $>10 \mathrm{~min}$ and to make $74 \mathrm{MHz}$ images of several radio sources. Fast frequency switching is also being considered as a standard calibration mode for the Atacama Large Millimeter Array (ALMA) in the future (D'Addario 2003).

\section{Observations}

We observed NGC 4261 on May 5, 2003 with the ten VLBA stations and we interleaved observations of $3 \mathrm{C} 273$ and 3C 279 to calibrate the inter-frequency offset and to test the technique on strong sources. Dynamic scheduling allowed us to observe during a period of good to excellent weather at most stations, using $256 \mathrm{Mbps}$ to record a bandwidth of $64 \mathrm{MHz}$ with 2-bit sampling. We recorded LCP only, the data were divided into 8 IFs with $8 \mathrm{MHz}$ bandwidth, each of which were subdivided into 64 spectral channels $125 \mathrm{kHz}$ wide. The correlator integration time was one second to allow monitoring of the phases with high time resolution. All antennas performed well, except for Fort Davis, where a receiver problem caused complete loss of $43 \mathrm{GHz}$ data. A summary of this observing run is given in Table 1.

In this paper, two integrations at $v_{\mathrm{r}}$ and $v_{\mathrm{t}}$ are called a "cycle", each integration of which is called a "half-cycle", and a sequence of cycles on the same source is called a "scan". Long (several minutes), continuous integrations on a single source at one frequency, e.g. fringe-finder observations, are also called "scans".

Several considerations influenced the experiment design.

\subsection{Frequency choice}

The target frequency should be an integer multiple of the reference frequency to avoid having to unwrap phase wraps. For example, if the frequency ratio, $r$, is the non-integer value of 2.5 , and the reference frequency phase wraps from $359^{\circ}$ to $0^{\circ}$, then the scaled target frequency phase will jump from $897.5^{\circ}\left(=177.5^{\circ}\right)$ to $0^{\circ}$, introducing a phase jump of $177.5^{\circ}$ into the calibration phase for a $1^{\circ}$ phase change at $v_{\mathrm{r}}$. In contrast, choosing $r=2.0$, when the reference frequency phase wraps from $359^{\circ}$ to $0^{\circ}$, the scaled target frequency phase changes from $718^{\circ}\left(=358^{\circ}\right)$ to $0^{\circ}$, corresponding to a change of $2^{\circ}$ for a $1^{\circ}$ phase change at $v_{\mathrm{r}}$. Hence, for integer values of $r$, phase wraps at the reference frequency introduce integer multiples of $360^{\circ}$ at the target frequency and so have no effect.

We chose a reference frequency of $14.375 \mathrm{GHz}$ since the third and sixth harmonics at $43.125 \mathrm{GHz}$ and $86.25 \mathrm{GHz}$ lie within the VLBA receiver bands. For convenience, we will refer to these frequencies as " $15 \mathrm{GHz}$ ", " $43 \mathrm{GHz}$ " and " $86 \mathrm{GHz}$ ", respectively. These frequencies should then be shifted slightly to end in $.49 \mathrm{MHz}$ or $.99 \mathrm{MHz}$ to allow correct operation of the VLBA pulse calibration detection system, and the final two digits should not change during the experiment as a change causes a time-consuming reconfiguration of the formatter. 
Table 1. Summary of observations May 5, 2003. The sources observed are in Col. (1), the scan duration is in Col. (2) and the frequency pairs in $\mathrm{GHz}$ are in Col. (3). An $a$ indicates fringe finder scans during which no frequency switching was done.

\begin{tabular}{|c|c|c|c|c|c|}
\hline Source & $\begin{array}{c}\text { Duration } \\
\text { (min) }\end{array}$ & $\begin{array}{l}\text { Freq. } \\
\text { pair }\end{array}$ & $\begin{array}{l}3 C 279 \\
3 C 273\end{array}$ & $\begin{array}{l}5 \\
5\end{array}$ & $\begin{array}{l}43 \\
43\end{array}$ \\
\hline (1) & (2) & (3) & $3 C 273$ & 5 & 1 \\
\hline OJ 287 & 10 & $15-86$ & NGC 4261 & 25 & $15-43$ \\
\hline OJ 287 & 10 & $15-43$ & $3 C 273$ & 5 & $15-43$ \\
\hline NGC 4261 & 25 & $15-43$ & $3 C 273$ & 5 & $15-86$ \\
\hline $3 \mathrm{C} 273$ & 5 & $15-43$ & NGC 4261 & 25 & $15-86$ \\
\hline NGC 4261 & 25 & $15-86$ & $3 C 279$ & 5 & $15-43$ \\
\hline $3 C 273$ & 5 & $15-86$ & $3 \mathrm{C} 279$ & 5 & $15-86$ \\
\hline \multirow[t]{2}{*}{$3 \mathrm{C} 273$} & 5 & $15-43$ & & & \\
\hline & & & $3 \mathrm{C} 273$ & 10 & $15-43$ \\
\hline OJ 287 & 5 & $15^{a}$ & & & $15-$ \\
\hline OJ 287 & 5 & $43^{a}$ & NGC 4261 & 25 & $15-43$ \\
\hline \multirow[t]{2}{*}{ OJ 287} & 5 & $86^{a}$ & $3 C 273$ & 5 & $15-43$ \\
\hline & & & $3 C 273$ & 5 & $15-86$ \\
\hline NGC 4261 & 25 & $15-43$ & NGC 4261 & 25 & $15-86$ \\
\hline $3 C 279$ & 5 & $15-43$ & $3 C 279$ & 5 & $15-86$ \\
\hline $3 C 279$ & 5 & $15-86$ & $3 C 279$ & 5 & $15-43$ \\
\hline $3 C 273$ & 5 & $15-86$ & & & \\
\hline $3 C 273$ & 5 & $15-43$ & $3 \mathrm{C} 279$ & 5 & $15^{a}$ \\
\hline NGC 4261 & 25 & $15-43$ & $3 \mathrm{C} 279$ & 5 & $43^{a}$ \\
\hline $3 \mathrm{C} 273$ & 5 & $15-43$ & $3 C 279$ & 5 & $86^{a}$ \\
\hline $3 C 273$ & 5 & $15-86$ & & & \\
\hline NGC 4261 & 25 & $15-86$ & $3 C 273$ & 5 & $15-43$ \\
\hline $3 C 279$ & 5 & $15-86$ & $3 C 273$ & 5 & $15-86$ \\
\hline \multirow[t]{2}{*}{$3 C 279$} & 5 & $15-43$ & NGC 4261 & 25 & $15-86$ \\
\hline & & & $3 C 273$ & 5 & $15-86$ \\
\hline \multirow[t]{2}{*}{$3 \mathrm{C} 273$} & 10 & $15-43$ & $3 C 273$ & 5 & $15-43$ \\
\hline & & & NGC 4261 & 25 & $15-43$ \\
\hline NGC 4261 & 25 & $15-43$ & $3 \mathrm{C} 279$ & 5 & $15-43$ \\
\hline $3 C 273$ & 5 & $15-43$ & $3 C 279$ & 5 & $15-86$ \\
\hline $3 C 273$ & 5 & $15-86$ & $3 C 273$ & 5 & $15-43$ \\
\hline NGC 4261 & 25 & $15-86$ & & & \\
\hline \multirow[t]{2}{*}{$3 \mathrm{C} 279$} & 5 & $15-86$ & $3 C 345$ & 5 & $15^{a}$ \\
\hline & & & $3 C 345$ & 5 & $43^{a}$ \\
\hline $3 \mathrm{C} 279$ & 5 & $15^{a}$ & $3 \mathrm{C} 345$ & 5 & $86^{a}$ \\
\hline $3 \mathrm{C} 279$ & 5 & $43^{a}$ & & & \\
\hline \multirow[t]{2}{*}{$3 \mathrm{C} 279$} & 5 & $86^{a}$ & $3 C 345$ & 10 & $15-43$ \\
\hline & & & $3 \mathrm{C} 345$ & 10 & $15-86$ \\
\hline
\end{tabular}

Thus, optimal frequencies are $14.37499 \mathrm{GHz}, 43.12499 \mathrm{GHz}$ and 86.24999 GHz.

\subsection{Integration times}

We chose a cycle time of $50 \mathrm{~s}$, of which $22 \mathrm{~s}$ were spent at the reference frequency of $15 \mathrm{GHz}$ and the remaining $28 \mathrm{~s}$ were spent at the target frequency, either $43 \mathrm{GHz}$ or $86 \mathrm{GHz}$. An average time of $7 \mathrm{~s}$ per half-cycle was lost in moving the subreflector between the feed horns, resulting in net integration times of $15 \mathrm{~s}$ at $v_{\mathrm{r}}$ and $21 \mathrm{~s}$ at $v_{\mathrm{t}}$. The integration times are a compromise, depending on source brightness, antenna sensitivity and expected weather conditions. This setup yielded a $5 \sigma$ detection limit of $89 \mathrm{mJy}$ in $15 \mathrm{~s}$ at $15 \mathrm{GHz}$ for the VLBA on a single baseline.

\subsection{Calibrator scans}

Calibrators must be observed frequently to monitor the phase offset $\Delta \Phi=\phi_{\mathrm{t}}^{\text {ins }}-r \tilde{\phi}_{\mathrm{r}}^{\text {ins }}$ between the two frequencies. A constraint which is important for core-shift measurements is that the calibrators must be achromatic, i.e., they must not have their own frequency-dependent core shifts. In the case of the observations presented here, uncalibrated ionospheric errors prevented such a measurement of $\Delta \Phi$. We used self-calibration with a long solution interval to calibrate the residual phase errors, and did not require measurement of the instrumental phase offset on calibrators.

As the core-shift information is not generally available, the best strategy is to observe at least two calibrators to test for a possible core shift in the calibrators. We included adjacent scans on two different calibrators to measure $\Delta \Phi$ before and after target source observations. Five of the calibrator scans were twice as long to provide more time for tests with strong signals.

\section{Data reduction}

\subsection{Standard steps}

Data reduction was carried out in AIPS. A calibration table entry was generated every $4.8 \mathrm{~s}$ to provide high temporal resolution. The amplitudes were calibrated using $T_{\text {sys }}$ and gain measurements provided by automatic noise-adding radiometry, and amplitude corrections for errors in the sampler thresholds were performed using autocorrelation data. Phase corrections for parallactic angles were applied (this step is not strictly required for the method to work) and a simple bandpass correction was derived at each frequency from one of the fringe finder scans.

The VLBA's pulse calibration system did not deliver data to calibrate the phase offsets between the IFs because the frequencies were changed too quickly. The pulse calibration system has a default integration time of $10 \mathrm{~s}$, plus one second for readout. If any part of that integration time is during a part of the scan that is flagged by the online system, then the integration is not accepted. Thus, one loses the first two integrations because the online system conservatively flags $10 \mathrm{~s}$ to $11 \mathrm{~s}$ after a frequency change, and there is an overlap between the flagged time and the second integration. During the whole experiment, only $\sim 10$ useful pulse-cal measurements were recorded per station at $15 \mathrm{GHz}$ (half-cycle time $22 \mathrm{~s}$ ), and $\sim 100$ at $43 \mathrm{GHz}$ (half-cycle time $28 \mathrm{~s}$ ), compared to the number of half-cycles at these frequencies of $\sim 630$ and $\sim 230$, respectively. We therefore used fringe-fitting on the same fringe finder scans as were used for bandpass calibration to correct for instrumental delays and inter-IF phase offsets. These offsets were found to be stable over the experiment. 


\subsection{Ionospheric correction}

Although the frequencies used in the project are quite high, ionospheric effects can not be neglected and will prevent a successful phase transfer if uncorrected. A typical ionospheric delay at a frequency of $v_{1}=100 \mathrm{MHz}$ is $0.1 \mu \mathrm{s}$ (10 turns of phase), but can be up to $10 \mu \mathrm{s}$. The exact number strongly depends on the time of day, time of year and time in the solar cycle. The delay scales with frequency as $v^{-2}$, and so for a delay at $100 \mathrm{MHz}$ of $0.1 \mu \mathrm{s}$, the ionospheric delays at $14.37499 \mathrm{GHz}$, $43.12499 \mathrm{GHz}$ and $86.24999 \mathrm{GHz}$ are $4.84 \mathrm{ps}, 0.54 \mathrm{ps}$ and $0.13 \mathrm{ps}$, respectively, corresponding to $25^{\circ}, 8.4^{\circ}$ and $4.0^{\circ}$ of phase. The linear phase versus frequency scaling law used by fast frequency switching cannot correct phase changes that are induced by the ionosphere. Ionospheric phase changes have much longer time-scales than tropospheric changes, and they can be calibrated before fringe-fitting when the electron content of the ionosphere along the line of sight is known. The AIPS task TECOR can use maps of ionospheric total electron content (TEC) derived from GPS data to calculate phase and delay corrections. Unfortunately, the error in these maps can be quite high, up to $20 \%$ when the TEC is as high as a few tens of TEC units ( 1 TEC unit equals $10^{16}$ electrons $\mathrm{m}^{-2}$ ), and up to $50 \%$ or higher when the TEC is of the order of a few TEC units. We have used the TEC maps produced by the Center for Orbit Determination in Europe $\left(\mathrm{CODE}^{2}\right)$ to calibrate the effects of the ionosphere. We found that these maps yielded slightly better results than those from the Jet Propulsion Laboratory (JPL), i.e., the residual phase errors after scaling were smaller. We do not know whether this finding is coincidental or whether the CODE maps generally give better results.

\subsection{Effect of frequency changes on the phases}

Amplitudes needed $2 \mathrm{~s}$ to $3 \mathrm{~s}$ longer to reach their final values during a frequency change than did the phases. Thus, the visibility phases are not very sensitive to even large errors in subreflector rotational position. The repeatability of the phases from cycle to cycle shows that positioning the subreflector along the optical axis is repeatable to $<5^{\circ}$ of phase at $15 \mathrm{GHz}$, which is much less than other sources of phase error in fast frequency switching. Six to seven seconds at the beginning of each half-cycle, when the subreflector was moved between feed horns, needed to be flagged.

\subsection{Phase solution and phase scaling}

We derived phase solutions at $15 \mathrm{GHz}$ using fringe-fitting, which is equivalent to self-calibration except that it also derives delay solutions. Its sensitivity therefore is not as good as self-cal, which performs a phase search only and is preferred. However, fringe-fitting yielded good results and a high detection rate in our experiment.

We fringe-fitted the $15 \mathrm{GHz}$ data using the AIPS task FRING, with an SNR threshold of 5 and delay and rate search windows of $20 \mathrm{~ns}$ and $50 \mathrm{mHz}$, respectively. As NGC 4261 has

\footnotetext{
${ }^{2}$ http://www.aiub.unibe.ch/ionosphere.html
}

an extended, double-sided jet structure at $15 \mathrm{GHz}$, we made a $15 \mathrm{GHz}$ image which we used as a source model in a second run of FRING, so that the phase solutions did not contain structural phase contributions. The solution interval was set to $1 \mathrm{~min}$, yielding one phase, delay and rate solution per half cycle. The detection rate was $\sim 90 \%$. The $15 \mathrm{GHz}$ solution (SN) table was written to a text file with TBOUT to do the phase scaling outside AIPS. We have written a Python program (FFSTG, the Fast Frequency Switching Table Generator) that processes an SN table in the following way.

First, a series of timestamps is generated from each pair of consecutive entries in the input table such that they coincide with the $v_{\mathrm{t}}$ half-cycles. Second, from each pair of consecutive $v_{\mathrm{r}}$ phase solutions, a solution is interpolated for the new timestamps consisting of a phase, a phase rate derived from the $v_{\mathrm{r}}$ phase solutions and the time interval between the $v_{\mathrm{r}}$ scans, and an interpolated delay. Both the phase rate and the delay do not need to be scaled by $r$ because the rate is stored in a frequency-independent format (in units of $\mathrm{s} \mathrm{s}^{-1}$ ) and the delay is non-dispersive. Third, the interpolated phase solution is scaled by the frequency ratio, $r$, yielding $v_{\mathrm{t}}$ phase solutions for the times at which the source was observed at $v_{\mathrm{t}}$.

We did not use the phase rates derived by fringe-fitting because each of those was derived from one half-cycle of $22 \mathrm{~s}$ length, whereas the phase rates interpolated from two consecutive half-cycles as described above used two half-cycles separated by $50 \mathrm{~s}$ and therefore have much better SNR. This requires the phase to change by less than $180^{\circ}$ in $50 \mathrm{~s}$, which was the case during our experiment due to sufficiently short half-cycles. A further advantage of deriving phase rates from pairs of phase solutions is that each $v_{\mathrm{r}}$ phase solution is used both in the determination of the phase rate to the preceding and the succeeding $v_{\mathrm{r}}$ solution, causing a smoothing of the phases with time and reducing the effect of outliers. Finally, the interpolated phase, phase rate and delay solutions are stored in an output table together with the $v_{\mathrm{t}}$ frequency ID. FFSTG provides an interface to Gnuplot to plot and inspect the input and output phases. One can select regions of interest and check the results of the scaling.

The table was imported to AIPS using TBIN and was used to update the most recent calibration table at the target frequency.

\section{Results}

\section{1. $43 \mathrm{GHz}$}

NGC 4261 was detected on most baselines at all times after scaling the $15 \mathrm{GHz}$ solutions to $43 \mathrm{GHz}$. Here, the term "detected" means that by inspecting the phase time series by eye one could see that the phase was not random. The correlated flux densities range from $30 \mathrm{mJy}$ on baselines of $800 \mathrm{M} \lambda$ to $160 \mathrm{mJy}$ on baselines of $30 \mathrm{M} \lambda$. The $43 \mathrm{GHz}$ half-cycle average visibilities on baselines to LA are shown in Figs. 2-4. The short-term fluctuations introduced by the troposphere are almost perfectly calibrated, but residual phase drifts remain on longer time-scales, especially at the beginning (before and 

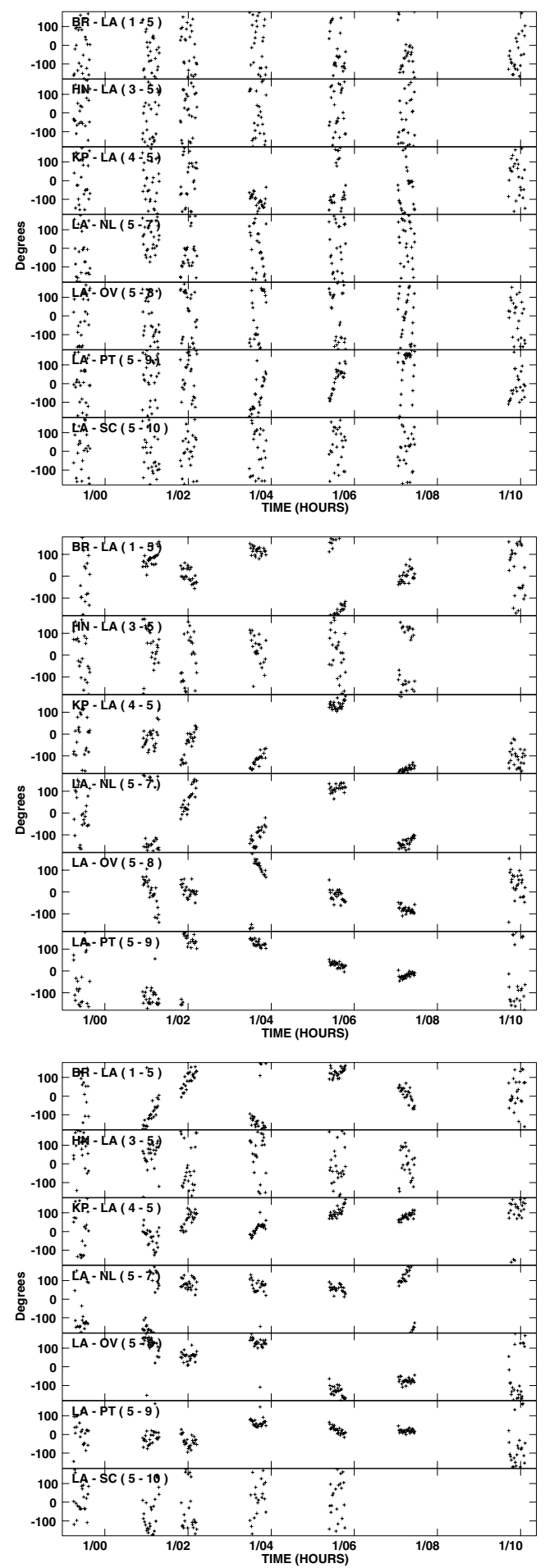

Fig. 2. Raw and calibrated visibility phases on baselines to Los Alamos. Each data point is an average over a half-cycle. Top: NGC 4261 raw $43 \mathrm{GHz}$ visibility phases on baselines to Los Alamos with only delay calibration applied. Middle: NGC 4261 calibrated $43 \mathrm{GHz}$ visibility phases on baselines to Los Alamos. Calibration used scaled-up phase solutions from fringe-fitting with a clean component model at $15 \mathrm{GHz}$, but no ionospheric correction has been applied. Bottom: NGC 4261 calibrated $43 \mathrm{GHz}$ visibility phases on baselines to Los Alamos. Calibration used ionospheric corrections and scaledup phase solutions from fringe-fitting with a clean component model at $15 \mathrm{GHz}$. The residual phase rates are much lower than in the middle panel.

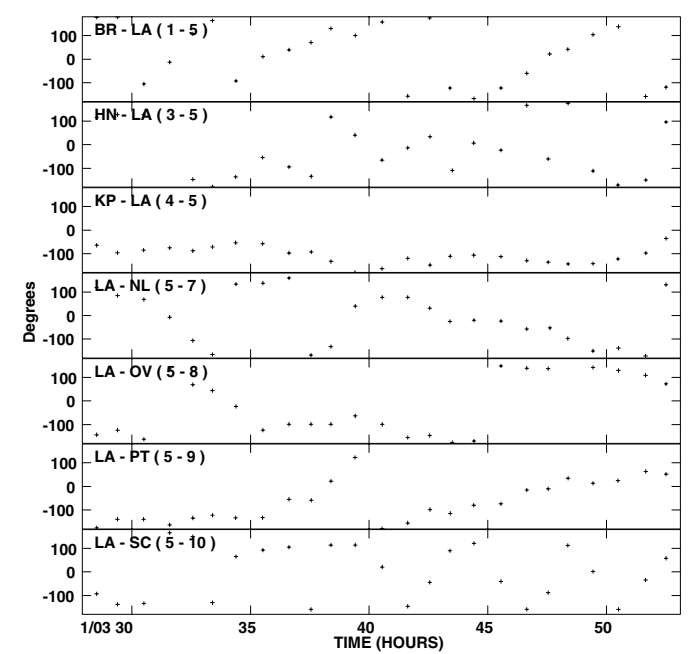

Fig. 3. Enlarged section of the top panel in Fig. 2.

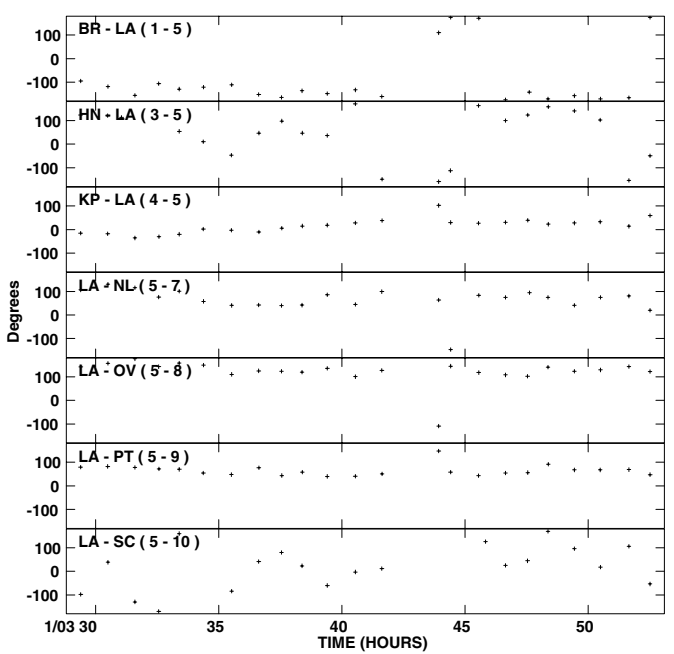

Fig. 4. Enlarged section of the bottom panel in Fig. 2.

during sunset) and at the end of the experiment, when the source elevation was lower.

\subsubsection{Structure functions}

Structure functions of the $43 \mathrm{GHz}$ visibility phases from a 25 min scan on NGC 4261 are shown in Fig. 5 showing the data at each stage of calibration. Phase wraps have been removed, allowing phase differences exceeding $360^{\circ}$.

The structure functions constructed from the calibrated phase time-series show a residual phase noise on the shortest time-scales $(50 \mathrm{~s})$ of $50^{\circ}$ early in the observations and of $33^{\circ}$ in the middle of the experiment (taking the median of the phase noise of all baselines). The phase noise increases toward longer time-scales, probably due to errors in the ionospheric models. Evidence for the long-term phase noise being dominated by ionosphere is as follows.

First, applying an ionospheric TEC correction significantly improved the coherence, as one can see by comparing the middle and bottom panel in Fig. 2. Second, the residual phase 


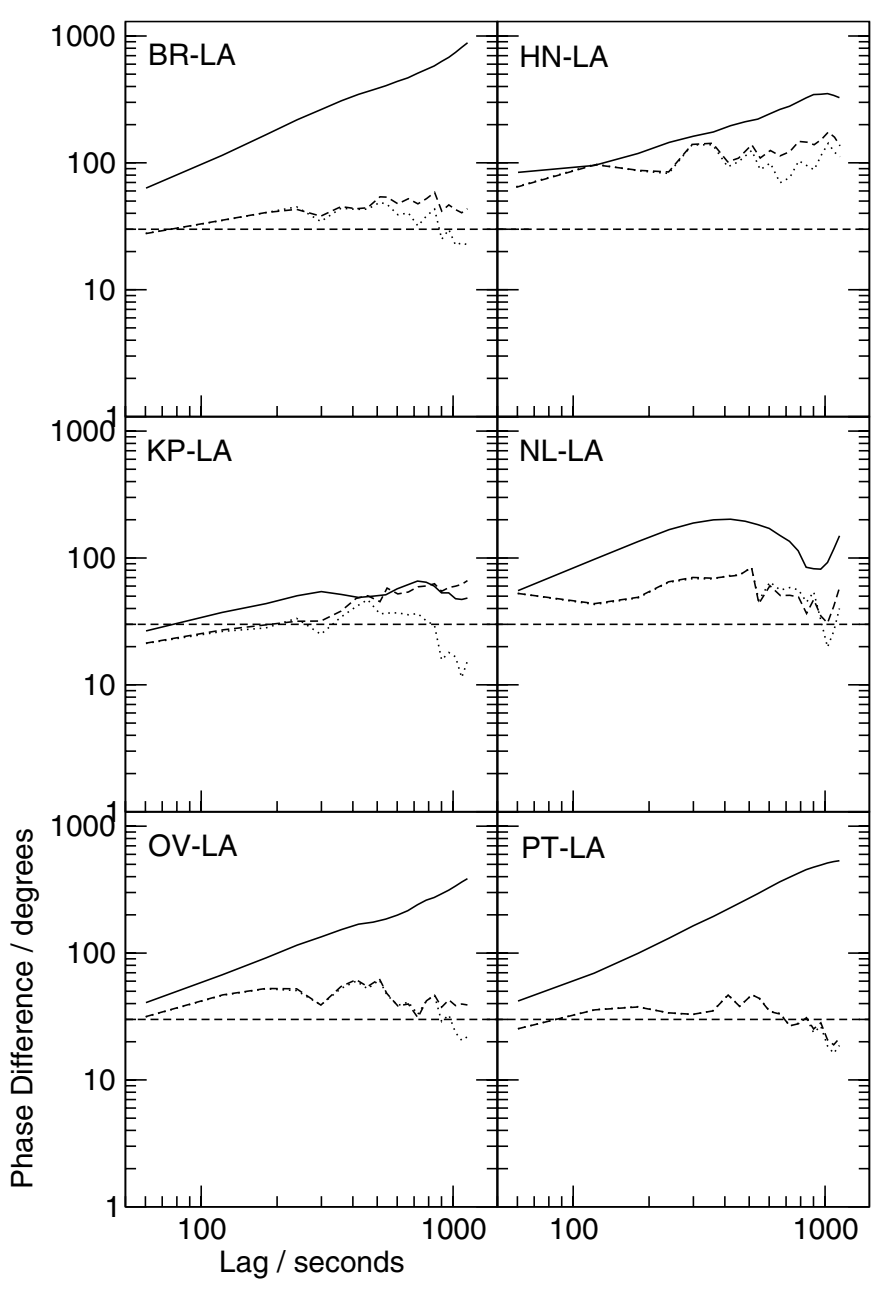

Fig. 5. Structure functions of the phase time series from $25 \mathrm{~min}$ of observation of NGC 4261 at $43 \mathrm{GHz}$. Solid lines show raw data observed in the middle of the experiment with delay calibration only. The data were averaged in frequency and over each half-cycle. Dashed lines show the same data calibrated with scaled-up phase solutions from fringe-fitting at $15 \mathrm{GHz}$, and dotted lines show the same data after fringe-fitting with a $30 \mathrm{~min}$ solution interval. Phase wraps have been removed. The horizontal dashed lines indicate the theoretical phase noise. The median residual phase noise of calibrated data with residual phase rates is $33^{\circ}$ on a $50 \mathrm{~s}$ time-scale, and without residual phase rates is $31^{\circ}$.

errors decreased in the middle of the experiment, when the average elevation of the source was $58^{\circ}$. During the first scan at 01:00 UT, the average source elevation at all antennas was $32^{\circ}$, hence the line of sight through the ionosphere was $63 \%$ longer, amplifying errors in the ionospheric model. Also, the experiment began briefly before sunset at the stations in the southwest US. This is a time of rapidly changing ionospheric TEC causing unstable phases early in the experiment on baselines to the south-western stations.

We fringe-fitted the $43 \mathrm{GHz}$ data calibrated with fast frequency switching with a solution interval of $30 \mathrm{~min}$ to remove the residual long-term phase drifts. The structure functions after applying this correction are shown as dotted lines in Fig. 5; the long-term phase noise is reduced as expected, and the phase noise on $50 \mathrm{~s}$ time scale is slightly lower, with a median of $44^{\circ}$ during the beginning and $31^{\circ}$ in the middle of the experiment.

\subsubsection{Expected phase noise}

The expected phase noise in the visibilities calibrated with fast frequency switching consists of five parts: (1) thermal phase noise at the reference frequency scaled by the frequency ratio; (2) thermal phase noise at the target frequency; (3) tropospheric phase changes during the two integrations; (4) ionospheric scintillations; and (5) errors in the source model at the reference frequency. Estimates of these contributions are as follows.

The $1 \sigma$ thermal noise on a baseline is given by

$\Delta S=\frac{1}{\eta_{\mathrm{s}}} \times \frac{S E F D}{\sqrt{2 \times \Delta v \times \tau}}$

(Walker 1995), where $\eta_{\mathrm{s}}$ is an efficiency factor (0.69 for the VLBA with 2-bit sampling as we used), SEFD is the antenna's system equivalent flux density in $\mathrm{Jy}, \Delta v$ is the bandwidth in $\mathrm{Hz}$ and $\tau$ the integration time in seconds.

The typical measured $S E F D$ for the VLBA antennas was $550 \mathrm{Jy}$ at $15 \mathrm{GHz}, 1436 \mathrm{Jy}$ at $43 \mathrm{GHz}$ and $5170 \mathrm{Jy}$ at $86 \mathrm{GHz}$ (at $43 \mathrm{GHz}$ and $86 \mathrm{GHz}$, these numbers are fairly variable between antennas). In our observations, $\tau$ was $15 \mathrm{~s}$ at $15 \mathrm{GHz}$ and $21 \mathrm{~s}$ at $43 \mathrm{GHz}$ and $86 \mathrm{GHz}$, and $\Delta v$ was $64 \mathrm{MHz}$. One therefore expects thermal noise levels of $18.2 \mathrm{mJy}, 40.1 \mathrm{mJy}$ and $145 \mathrm{mJy}$ at $15 \mathrm{GHz}, 43 \mathrm{GHz}$ and $86 \mathrm{GHz}$, respectively. At $15 \mathrm{GHz}$, NGC 4261 has a correlated flux density, $S$, between $60 \mathrm{mJy}$ and $300 \mathrm{mJy}$, depending on baseline length, so we adopt either $80 \mathrm{mJy}$, representative of long baselines, or $200 \mathrm{mJy}$, representative of short baselines (the results of which we give in brackets).

(1) On a single baseline, the expected SNR of a detection at $15 \mathrm{GHz}$ is $S / \Delta S=80 \mathrm{mJy} / 18.2 \mathrm{mJy}=4.61$ (11.0) when averaging over the band. Fringe-fitting, however, uses all baselines to one particular antenna to derive a phase correction, and so the SNR is increased by $\sqrt{N}$, where $N$ is the number of baselines. $N \approx 8$, so the SNR of a detection at $15 \mathrm{GHz}$ increases to 12.4 (31.1). We have converted the SNR into a visibility phase rms using the following approach. Let us assume that the phase of the true visibility is 0 . The probability distribution of the measured visibility phase as a function of SNR, $p(\phi)$, is given in Eq. (6.63b) in Thompson et al. (2001). The square of the phase rms derived from that probability is:

$\sigma^{2}=\frac{\int \phi^{2} p(\phi) \mathrm{d} \phi}{\int p(\phi) \mathrm{d} \phi}$

We have evaluated the integral numerically and find a phase rms of $4.61^{\circ}\left(1.84^{\circ}\right)$. This phase noise is scaled by the frequency ratio to $13.8^{\circ}\left(5.53^{\circ}\right)$ at $43 \mathrm{GHz}$ and $27.6^{\circ}\left(11.1^{\circ}\right)$ at $86 \mathrm{GHz}$.

(2) Assuming that NGC 4261 has a compact flux density of $100 \mathrm{mJy}$ at both $43 \mathrm{GHz}$ and $86 \mathrm{GHz}$, the thermal noise contributions according to Eq. (6) are $26.3^{\circ}$ and $76.0^{\circ}$, respectively. 

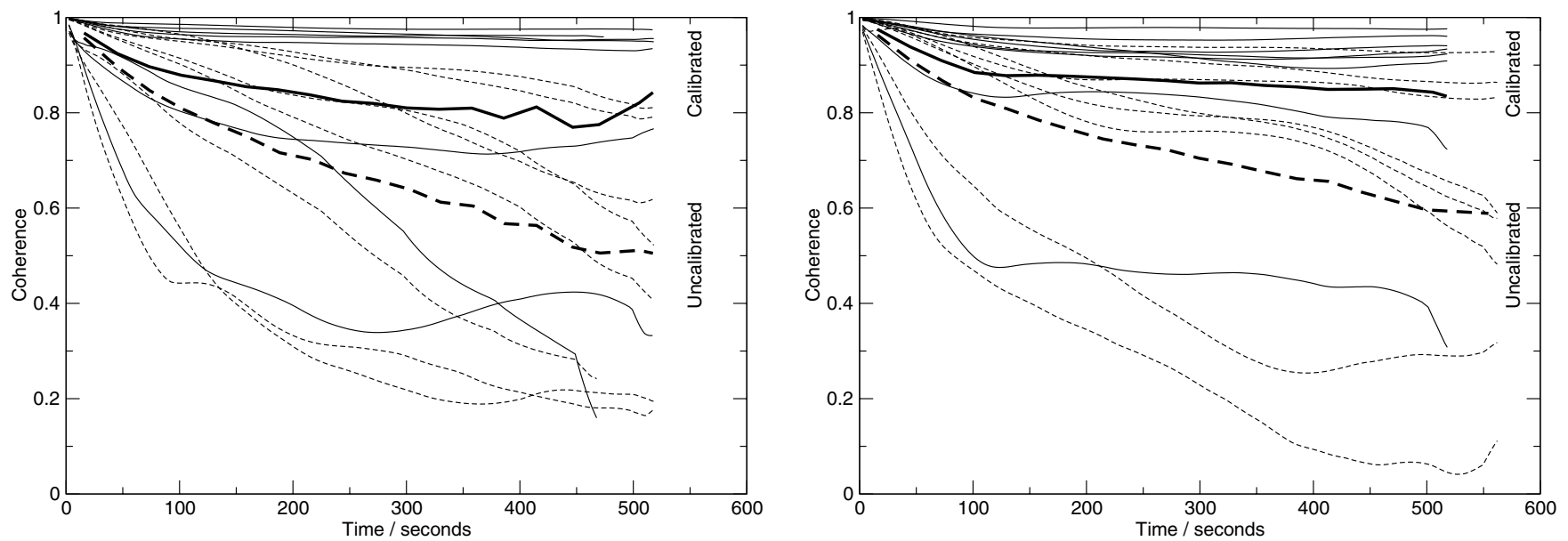

Fig. 6. Coherence diagrams showing the improvement achieved at $43 \mathrm{GHz}$ using fast frequency switching. The vector sum over progressively longer time intervals has been computed on all baselines and has been normalized by the scalar sum of the vectors, after setting the phases to zero. Bold lines show the average over all baselines in bins of $28 \mathrm{~s}$, i.e., the length of one half-cycle. The coherence was analysed using visibilities on baselines to Kitt Peak, which had moderate to good (but not excellent) weather conditions, and hence is representative for the array performance during the observing run. Shown are 3C 273 data observed in the beginning (centred on 03:20 UT, left panel) and the middle (centred on 06:50 UT, right panel) of the experiment at $43 \mathrm{GHz}$ with interleaved $15 \mathrm{GHz}$ observations for calibration. $43 \mathrm{GHz}$ data from $10 \mathrm{~min}$ scans with (solid lines) and without (dashed lines) phase calibration from $15 \mathrm{GHz}$ have been averaged in frequency. As $3 \mathrm{C} 273$ is strong, the data are essentially free of thermal noise and show the coherence improvement with fast frequency switching.

Adding those in quadrature to the scaled-up thermal rms phase noise from $15 \mathrm{GHz}$ yields $29.7^{\circ}\left(26.9^{\circ}\right)$ at $43 \mathrm{GHz}$ and $80.9^{\circ}$ $\left(76.8^{\circ}\right)$ at $86 \mathrm{GHz}$.

(3) We estimated the tropospheric phase noise within the switching cycle time using structure functions of $3 \mathrm{C} 273$. On

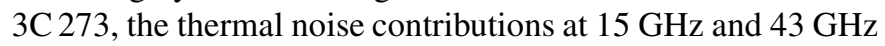
are $0.04^{\circ}$ and $0.4^{\circ}$, respectively, so that the visibility phases are essentially free of thermal noise and any phase changes during and between the half-cycles are due to changes in the troposphere. We found the median rms phase noise on a $50 \mathrm{~s}$ time-scale after fringe-fitting with a 30 min solution interval to remove the residual long-term phase drift to be $13.3^{\circ}$ at $43 \mathrm{GHz}$, or $26.6^{\circ}$ at $86 \mathrm{GHz}$.

The amplitudes of (4) (ionospheric scintillations) were found to be less than three radians at $378 \mathrm{MHz}$ on time-scales up to $40 \mathrm{~s}$ by Yeh \& Liu (1982). When scaled to $15 \mathrm{GHz}$, they contribute only $0.1^{\circ}$ on time-scales of $40 \mathrm{~s}$, and even less at the higher frequencies, so their contribution remains miniscule and is no longer considered. The contribution of (5) (errors in the source model) is also not significant. First, these errors do not contribute a noise-like component but rather long-term phase errors similar to errors in the TEC model. Second, phase corrections at $15 \mathrm{GHz}$ to account for the source structure were at most $50^{\circ}$ in $6 \mathrm{~h}$, or $3.5^{\circ}$ in $25 \mathrm{~min}$, and only on the longest baselines. This means that introducing a source model at all required changes of order a few degrees per $30 \mathrm{~min}$. Errors in the model, however, are expected to be much smaller because the sampling of the $(u, v)$ plane at $15 \mathrm{GHz}$ was excellent. They should barely exceed $1^{\circ}$ at $15 \mathrm{GHz}$, or $3^{\circ}$ at $43 \mathrm{GHz}$. This is much less than all other source of phase noise, and can therefore be neglected.
Adding the first three non-negligible noise components in quadrature yields $32.6^{\circ}\left(30.0^{\circ}\right)$ at $43 \mathrm{GHz}$ and is in excellent agreement with the measured $\mathrm{rms}$ phase noise of $31^{\circ}$.

\subsubsection{Coherence}

Another way of illustrating the performance of the corrections is with coherence diagrams (Fig. 6). For the plots shown, 3C $27343 \mathrm{GHz}$ data from all baselines to KP have been used. All data were calibrated using fast frequency switching (no subsequent fringe-fitting), and were averaged over the band and in time to obtain one visibility per baseline and half-cycle. The vector average of these visibilities has been computed over progressively longer time intervals and normalized by the scalar average.

The left panels show data from a 25 min scan centred on 03:20 UT and the right panels show data from a 25 min scan centred on 06:50 UT. Bold lines show the average over all baselines. The coherence achieved in the middle of the experiment with fast frequency switching is a little better than that achieved during the beginning of the observations.

\subsubsection{Stability of the phase offset}

After correction of the ionopheric phase errors, the visibility phases on the LA-PT baseline were stable to $<1$ rad over $10 \mathrm{~h}$ (bottom panel of Fig. 2). This indicates that the instrumental phase offset, $\Delta \Phi$, was also stable within the same limits, and it appears to be sufficient to determine $\Delta \Phi$ a few times throughout the experiment. 


\subsubsection{Imaging}

Before making an image from the $43 \mathrm{GHz}$ visibilities calibrated with fast frequency switching, the residual phase offsets and phase rates were removed using fringe-fitting with a solution interval of $30 \mathrm{~min}$ so that one solution per scan was obtained. The resulting dirty image (Fig. 7) has a peak flux density of $79 \mathrm{mJy}^{-1}$ beam $^{-1}$ and an rms noise of $4.4 \mathrm{mJy}^{\text {beam }}{ }^{-1}$, yielding a dynamic range of 18:1. After several cycles of phase self-calibration with a solution interval of $30 \mathrm{~s}$ and one cycle of amplitude self-calibration with a solution interval of $12 \mathrm{~h}$, the peak flux density was $95 \mathrm{mJy}^{\mathrm{beam}}{ }^{-1}$ and the rms noise was $0.78 \mathrm{mJy}$ beam $^{-1}$, so the dynamic range improved to $122: 1$ (Fig. 8). The theoretical rms noise at $43 \mathrm{GHz}$ was expected to be $0.46 \mathrm{mJy}_{\text {beam }}{ }^{-1}$. Unfortunately, the ionospheric phase errors and the need to fringe-fit to calibrate them did not allow a core shift measurement to be made.

Our final image has a synthesized beam size of 0.42 mas $\times$ 0.25 mas, corresponding to a linear resolution of $720 R_{\mathrm{s}}$. This is about $50 \%$ larger than achieved by Jones et al. (2000) with conventional, non-phase-referencing $43 \mathrm{GHz}$ VLBA observations. The difference in resolution is due to two factors. First, we made only few detections on baselines to Mauna Kea. This is because Mauna Kea was observing at predominantly low elevations, hence suffering more from ionospheric phase errors. Second, we used natural weighting to increase sensitivity to extended structures, whereas Jones et al. (2000) used uniform weighting for increased resolution.

Notwithstanding these differences, our $43 \mathrm{GHz}$ image is very similar to that published as Fig. 5 in Jones et al. (2000). Both observations show a dominant core and a jet extending 1.4 mas to the west, with indications of emission at 2 mas west of the core. Both images indicate the presence of a counterjet extending up to 1 mas east of the core. However, Jones et al. (2000) find a peak flux density of $141 \mathrm{mJy}$ beam $^{-1}$, whereas we find a peak flux density of $95 \mathrm{mJy}^{\text {beam }}{ }^{-1}$. The observations were separated by $5.7 \mathrm{yr}$, and source variability may have caused some (or all) of the difference. Furthermore, changing tropospheric opacity during either observation may have affected the peak flux density. In our image, the eastern jet looks smooth, whereas it shows components in the observation of Jones et al. (2000). This probably arises from the different weighting schemes and image fidelity limits, rather than from a real change of jet character.

\section{2. $86 \mathrm{GHz}$}

Following the same data reduction path as for the $43 \mathrm{GHz}$ data, we obtained good detections of NGC 4261 at $86 \mathrm{GHz}$ on baselines among the four stations FD, KP, LA, and PT and only weak detections on baselines to NL, OV and MK. Again, a "detection" means that in a plot of visibility phase with time as in Fig. 9, one can see that the phases cluster and are not random.

Uncorrected and corrected visibility phases are plotted in Fig. 9. The improvement in coherence is clearly visible as a pronounced clustering of points in the lower panel, compared to quasi-random phases in the upper panel. Structure functions and coherence plots from a 25 min scan of NGC 4261

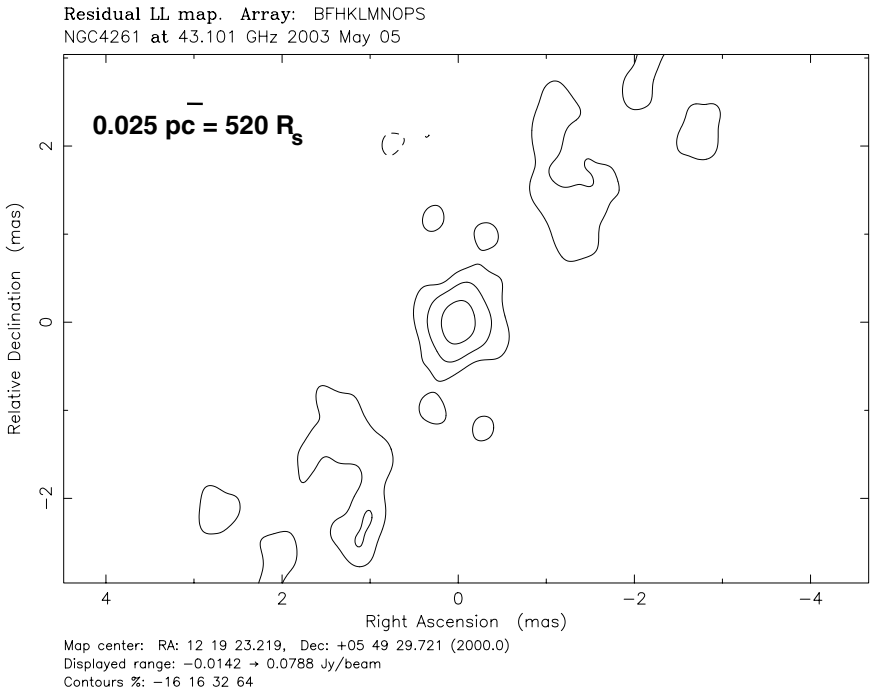

Fig. 7. Naturally weighted, full-resolution dirty image of NGC 4261 at $43 \mathrm{GHz}$, calibrated with scaled-up phase solutions from $15 \mathrm{GHz}$. Fringe-fitting has been used to solve for one residual phase and rate solution per 25 min scan before exporting the data to Difmap. No further self-calibration has been applied. The image noise is $4.4 \mathrm{mJy}$ beam $^{-1}$ and the dynamic range is $18: 1$. The synthesized beam size is 0.38 mas $\times 0.18$ mas. The bar in the upper left corner shows the size of the minor axis.

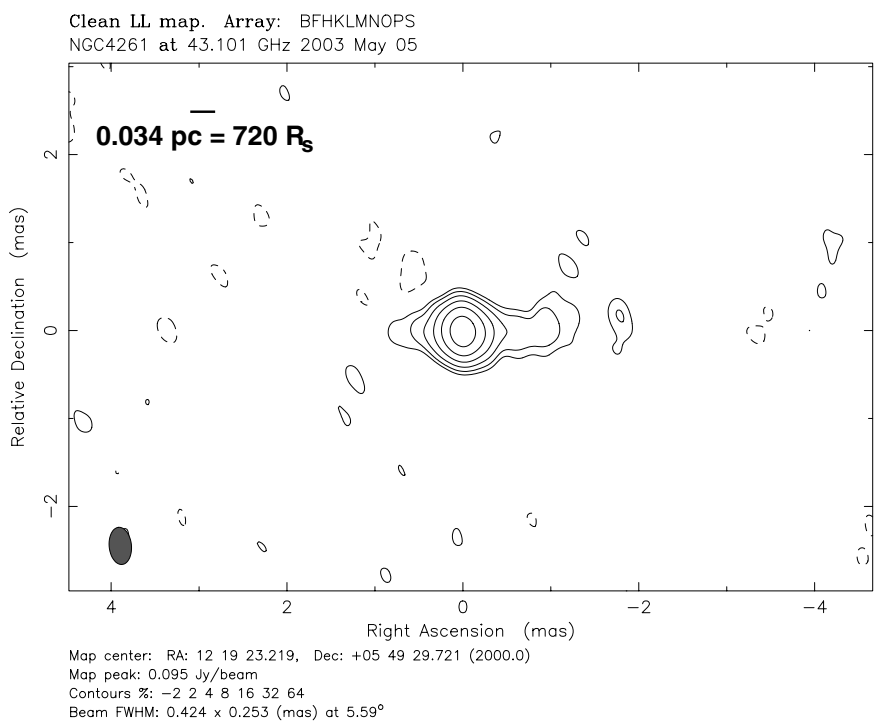

Fig. 8. Data and imaging parameters as in Fig. 7, but several cycles of phase self-calibration with a solution interval of $30 \mathrm{~s}$ and one cycle of amplitude self-calibration with a solution interval of $12 \mathrm{~h}$ have been applied. The final image was cleaned and has an rms noise of $0.78 \mathrm{mJy}$ beam $^{-1}$ and a dynamic range of $122: 1$. The synthesized beam size is 0.42 mas $\times 0.25$ mas. The bar in the upper left corner shows the size of the minor axis.

are shown in Figs. 10 and 11. The coherence improvement is especially pronounced on the FD-LA baseline (upper left panel in Fig. 10), whereas the improvement on the PT-LA and OV-LA baselines is small. The coherence plot in Fig. 11 shows 

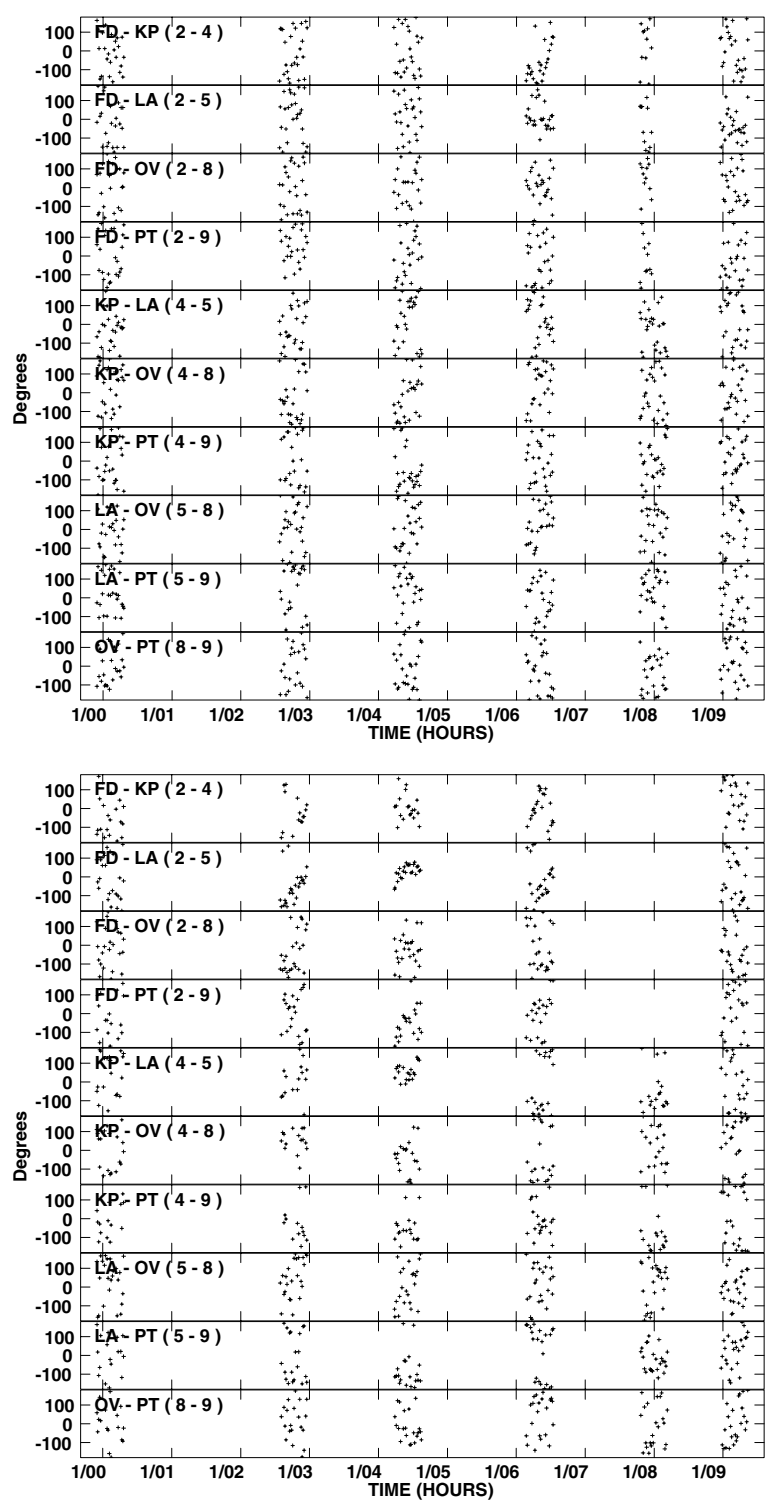

Fig. 9. $86 \mathrm{GHz}$ visibility phases. Top: NGC $4261 \mathrm{raw} 86 \mathrm{GHz}$ visibility phases on baselines among Fort Davis, Kitt Peak, Los Alamos, Owens Valley and Pie Town with only delay calibration applied. Bottom: $86 \mathrm{GHz}$ visibility phases on baselines among Fort Davis, Kitt Peak, Los Alamos, Owens Valley and Pie Town. Calibration has been done with scaled-up phase solutions from fringe-fitting at $15 \mathrm{GHz}$ using a clean component model. Good detections were made during almost every 25 min scan observed at night between 2:00 UT and 7:00 UT, when the source elevation was high and ionospheric effects lower.

that the coherence on baselines to KP increased from $35 \%$ to almost $60 \%$ on time-scales of $20 \mathrm{~min}$.

A dirty image is shown in Fig. 12 and a cleaned image in Fig. 13. The peak flux density is $59.3 \mathrm{mJy}$. This is the first detection of NGC 4261 with VLBI at $86 \mathrm{GHz}$. It is also probably the weakest continuum object ever detected with VLBI at this frequency. The next brightest detection of which we are aware is $85 \mathrm{mJy}$ in the $86 \mathrm{GHz}$ image of $1308+328$ by Porcas \& Rioja (2002), using conventional phase referencing to a calibrator $14.3^{\prime}$ away. With only delay calibration applied, the median

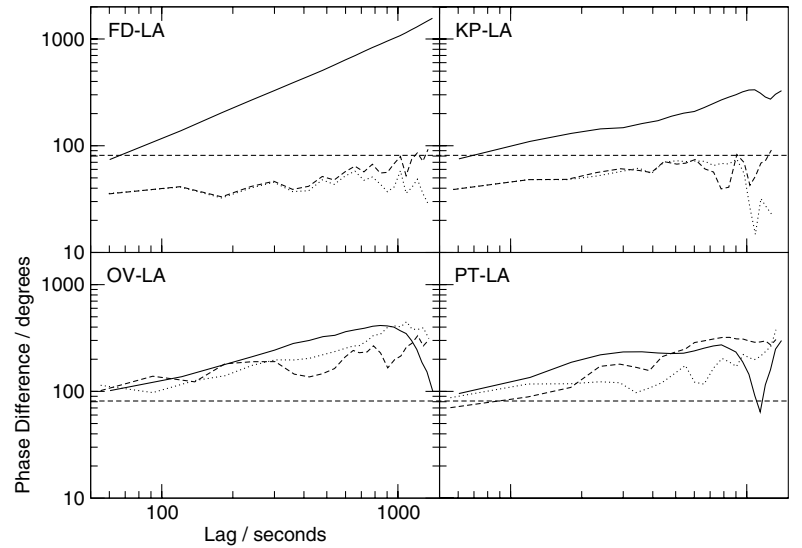

Fig. 10. Structure functions of a $25 \mathrm{~min}$ scan at $86 \mathrm{GHz}$, showing the same stages of calibration as in Fig. 5. Note that the expected noise level, indicated by the horizontal dashed lines, is a guideline only. It depends on the correlated source flux density which varies with baseline length and orientation (see Sect. 5.2).

\section{Coherence on baselines to KP}

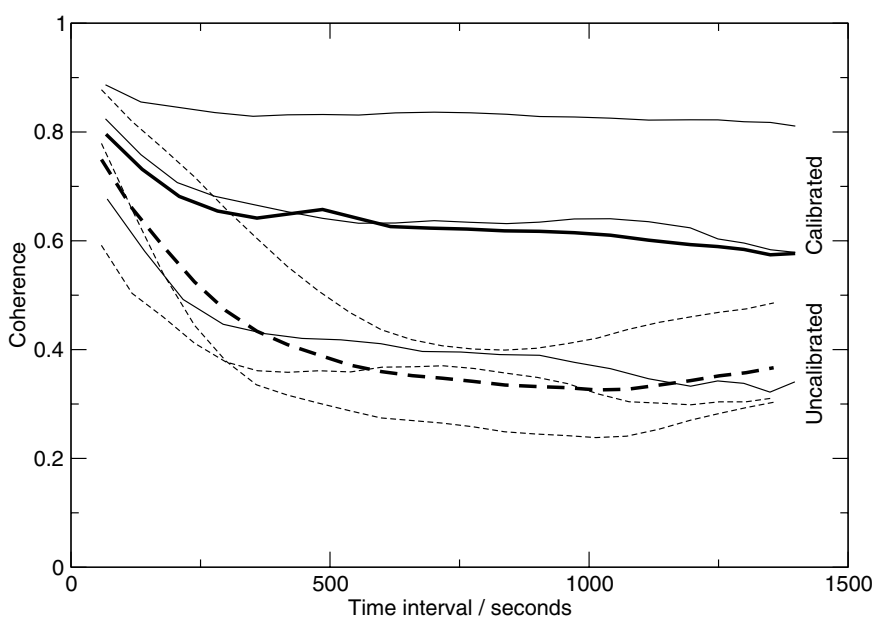

Fig. 11. The coherence improvement at $86 \mathrm{GHz}$ with fast frequency switching on baselines to KP, obtained in the same way as for Fig. 6 . However, we did not observe any calibrator long enough to generate coherence plots, so the diagram was constructed from NGC 4261 data. The coherence therefore is significantly lowered by thermal noise.

rms phase noise of the best 25 min scan is $104^{\circ}$, after applying the scaled $15 \mathrm{GHz}$ phase solutions is $70^{\circ}$ and after fringefitting with a $30 \mathrm{~min}$ solution interval is $80^{\circ}$. The increase in rms phase noise after removal of phase rates is unexpected and has an unknown cause.

The expected thermal phase noise is the quadrature sum of the scaled-up $15 \mathrm{GHz}$ noise and the $86 \mathrm{GHz}$ noise, the former of which we estimated to be $27.6^{\circ}$ on long and $11.1^{\circ}$ on short baselines, and the latter, assuming a correlated flux density of $100 \mathrm{mJy}$ and using Eq. (6), is $76.0^{\circ}$. The quadrature sum is $80.9^{\circ}$ on long and $76.8^{\circ}$ on short baselines, and adding the tropospheric phase errors of $26.6^{\circ}$ is $85.1^{\circ}$ and $81.3^{\circ}$, in agreement with the measured noise levels. 
Residual LL map. Array: BFHKLMNOPS NGC4261 at 86.226 GHz 2003 May 06

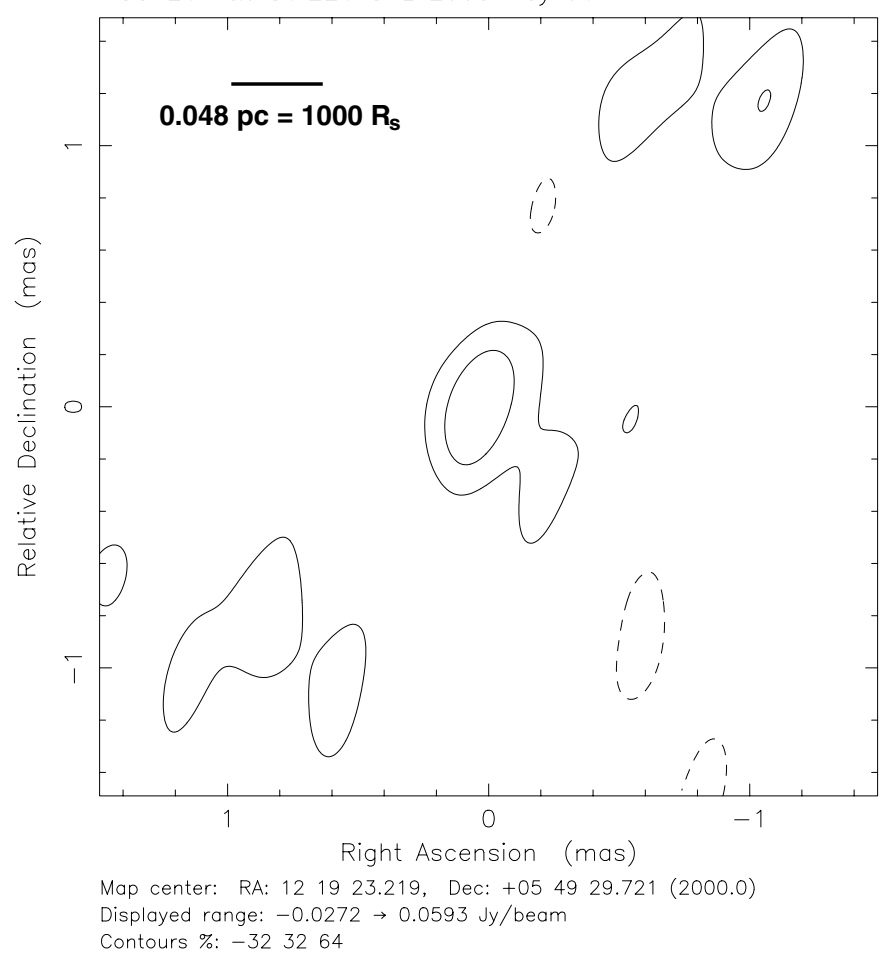

Fig. 12. Naturally weighted, full-resolution dirty image of NGC 4261 at $86 \mathrm{GHz}$, calibrated with scaled-up phase solutions from $15 \mathrm{GHz}$. Fringe-fitting has been used to solve for one residual phase and rate solution per 25 min scan before exporting the data to Difmap. No further self-calibration and no deconvolution has been applied. The image noise is $8.4 \mathrm{mJy}^{\text {beam }}{ }^{-1}$ and the dynamic range is $7: 1$. The synthesized beam has a diameter of 0.35 mas $\times 0.54$ mas in PA $11^{\circ}$ and corresponds to a linear resolution of $0.048 \mathrm{pc}$ or $1000 R_{\mathrm{s}}$. The bar in the upper left corner shows the size of the minor axis.

Note that these numbers provide a guideline only. The largest contribution to the expected $86 \mathrm{GHz}$ noise is the $76.0^{\circ}$ from thermal noise in the $86 \mathrm{GHz}$ receivers. This number depends on the source flux density which is a strong function of baseline length and orientation. For example, the average visibility amplitude on the relatively short FD-LA baseline was $180 \mathrm{mJy}$ at 4:30 UT (not $100 \mathrm{mJy}$ as adopted above), reducing the $86 \mathrm{GHz}$ thermal noise to $55.2^{\circ}$. The expected total phase noise, indicated by the dashed horizontal line in the upper left panel in Fig. 10, then drops to $62.3^{\circ}$.

The rms noise in the final $86 \mathrm{GHz}$ image is $7.4 \mathrm{mJy} \mathrm{beam}^{-1}$, compared to an expected thermal noise of $3.7 \mathrm{mJy}^{\text {beam }}{ }^{-1}$. The dynamic range in the image is $7: 1$.

\subsection{Discussion of the $86 \mathrm{GHz}$ image}

The final $86 \mathrm{GHz}$ image of NGC 4261 in Fig. 13 has a synthesized beam size of 0.28 mas $\times 0.52$ mas in PA $19^{\circ}$, which is about $12 \%$ larger than the clean beam of the $43 \mathrm{GHz}$ image. This is mostly due to the lack of detections on long baselines and the phase noise arising from low elevations at Mauna Kea (see Sect. 5.1.5) was larger by a factor of two at $86 \mathrm{GHz}$ than at $43 \mathrm{GHz}$ due to the higher frequency. The VLBA station at

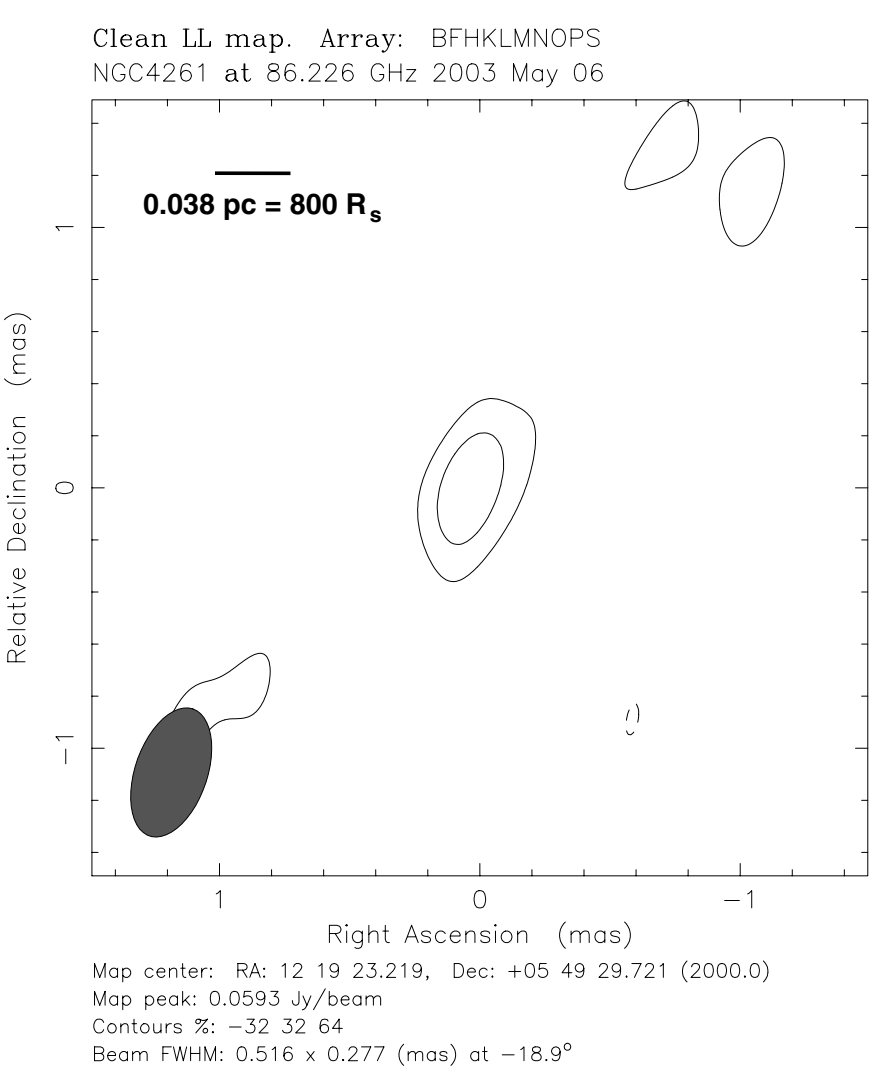

Fig. 13. Naturally weighted, full-resolution clean image of NGC 4261 at $86 \mathrm{GHz}$ using the same data and calibration as in Fig. 12. No further self-calibration has been applied. The image noise is $7.5 \mathrm{mJy}^{\text {beam }}{ }^{-1}$ and the dynamic range is $8: 1$. The synthesized beam has a diameter of 0.28 mas $\times 0.52$ mas in PA $19^{\circ}$ and corresponds to a linear resolution of 0.038 pc or $800 R_{\mathrm{s}}$.

Hancock was being commissioned at $86 \mathrm{GHz}$ and the station at Brewester was not equipped at $86 \mathrm{GHz}$; both would have contributed long baselines at this frequency. Also, the complete loss of $43 \mathrm{GHz}$ data at Fort Davis resulted in poorer sampling of short $(u, v)$ spacings at $43 \mathrm{GHz}$ but not at $86 \mathrm{GHz}$, so there is an emphasis on long baselines at $43 \mathrm{GHz}$ and on short baselines at $86 \mathrm{GHz}$. The resolution at $86 \mathrm{GHz}$ corresponds to $0.038 \mathrm{pc}$, or $800 R_{\mathrm{s}}$, at the distance of NGC 4261 .

The image displays a point source only; further selfcalibration of the data did not reliably converge on any extended structure. The point source is probably the brightest component seen in the $43 \mathrm{GHz}$ image, which probably corresponds to the jet base. Our observations therefore did not allow us to resolve the jet collimation region. However, we are confident that the suggested improvements to the observing strategy to calibrate ionospheric phase errors can significantly improve the detection rates on long baselines and hence increase the resolution.

\section{Benefits of fast frequency switching}

The primary use of fast frequency switching is to detect sources that are too weak for self-calibration at the target frequency within the atmospheric coherence time, but can be reliably detected at a lower frequency. The $5 \sigma$ detection limit of the 
VLBA at $43 \mathrm{GHz}$ within $120 \mathrm{~s}$, with $64 \mathrm{MHz}$ bandwidth and 2-bit sampling, and neglecting coherence loss due to tropospheric phase changes, is $84.0 \mathrm{mJy}$ (i.e., a thermal noise level of $16.8 \mathrm{mJy}$ on a single baseline). The $1 \sigma$ noise level using fast frequency switching with half-cycle times of $22 \mathrm{~s}$ at $15 \mathrm{GHz}$ and $28 \mathrm{~s}$ at $43 \mathrm{GHz}$ (yielding net integration times of $15 \mathrm{~s}$ and $21 \mathrm{~s}$, respectively), after one cycle is $67.7 \mathrm{mJy}$, of which $40.1 \mathrm{mJy}$ is thermal noise in the raw $43 \mathrm{GHz}$ visibility, $54.6 \mathrm{mJy}$ is thermal noise in the phase solutions after scaling from $15 \mathrm{GHz}$, and neglecting noise due to tropospheric phase changes. The $1 \sigma$ noise level after $120 \mathrm{~s}$ of fast frequency switching (i.e., 2.4 cycles) is $43.7 \mathrm{mJy}$. The noise level of fast frequency switching reaches that of a conventional $120 \mathrm{~s}$ integration at $43 \mathrm{GHz}$ of $16.8 \mathrm{mJy}$ after $812 \mathrm{~s}$ (13.5 min). Any longer integration with fast frequency switching then yields detection thresholds that are out of reach with conventional methods.

Furthermore, the proposed application of fast frequency switching to detect core shifts in AGN can supplement jet physics with observational constraints which are otherwise difficult to obtain.

Fast frequency switching is limited by the source strength at the reference frequency, and $100 \mathrm{mJy}$ at $15 \mathrm{GHz}$ is, from our experience, a reasonable minimum required for a successful observation. Many sources exist that meet this criterion. The 130 sources in the $2 \mathrm{~cm}$ VLBI observations by Kellermann et al. (1998), for example, have peak flux densities of $100 \mathrm{mJy} \mathrm{beam}^{-1}$ or more; all of them can be observed at $86 \mathrm{GHz}$ using fast frequency switching.

Another estimate of the number of new sources made accessible to $86 \mathrm{GHz}$ VLBI by fast frequency switching can be made by means of the $\log N-\log S$ relation. Let us assume that the sources observable with conventional $86 \mathrm{GHz}$ VLBI are distributed homogeneously in an Euclidean universe, so that lowering the detection limit does not yield the detection of a new population. The number of sources above a given flux-density limit, $S$, then increases as $S^{-1.5}$. We further make the conservative assumption that these sources have $\alpha=0$ between $15 \mathrm{GHz}$ and $86 \mathrm{GHz}$, because they are compact.

The current $5 \sigma$ detection limit of the VLBA at $86 \mathrm{GHz}$ is $605 \mathrm{mJy}$ (five times the thermal noise of a $30 \mathrm{~s}$ integration with $64 \mathrm{MHz}$ bandwidth and 2-bit sampling on a single baseline). In fringe-fitting, all $N$ antennas are used to derive a phase solution, so the noise level is reduced by $\sqrt{N}$, and with $N=8$, the $5 \sigma$ detection limit is $214 \mathrm{mJy}$. Fast frequency switching allows one to observe any compact source with $S_{86 \mathrm{GHz}} \geq 100 \mathrm{mJy}$, a factor of 2.14 fainter than with conventional methods, and one expects 3.1 times as many sources brighter than $100 \mathrm{mJy}$ than there are brighter than $214 \mathrm{mJy}$, following the $\log N-\log S$ relation. A little less conservative estimate would be to allow for spectral indices as steep as $\alpha=-0.3$, so that sources with $S_{15 \mathrm{GHz}}=100 \mathrm{mJy}$ have $S_{86 \mathrm{GHz}}>60 \mathrm{mJy}$. The number of observable sources would then increase by a factor of 6.7 times the number accessible to conventional techniques.

The absolute number of presently observable sources is much more difficult to determine due to selection effects in the various radio source catalogues. However, we can make a simple estimate using the recent $86 \mathrm{GHz}$ VLBI survey by
Lobanov et al. (2002). They aimed at observing more than 100 sources selected from the literature that had an expected compact flux density of $S_{86 \mathrm{GHz}}>300 \mathrm{mJy}$. Applying the fast frequency switching sensitivity estimates then yields between 520 and 1100 sources observable with the VLBA at $86 \mathrm{GHz}$.

\section{Summary}

1. Fast frequency switching can be used to calibrate tropospheric phase fluctuations if the switching cycle time is shorter than the atmospheric coherence time. The accuracy is limited by tropospheric phase changes between and during the reference frequency integrations.

2. Insufficient knowledge about the ionosphere's total electron content (TEC) has prevented calibration of the inter-band phase offset and hence it was not possible to make a pure phase-referenced image without using self-calibration. This also prevented the detection of a core shift. Current global TEC models derived from GPS data have errors that are too large to sufficiently calibrate the ionospheric component of the phase changes. A possible solution is to insert frequent (every $10 \mathrm{~min}$ ) scans in the $1.4 \mathrm{GHz}$ band with widely separated IF frequencies to derive the ionospheric delay on the line of sight to the target source.

3. On the most stable baseline between LA and PT, the instrumental phase offset was stable to $<1$ rad over $10 \mathrm{~h}$. It therefore seems to be sufficient to determine the offset a few times throughout the experiment.

4. Truly simultaneous observations at two bands would reduce the residual phase errors even further because tropospheric phase changes within the switching cycle time would be entirely removed.

5. At $86 \mathrm{GHz}, \mathrm{NGC} 4261$ was detected as a point source with $59.3 \mathrm{mJy} \mathrm{beam}^{-1}$ flux density and so is the weakest source ever detected with VLBI at that frequency. Our observation at $43 \mathrm{GHz}$ yielded a resolution corresponding to $720 R_{\mathrm{s}}$ and hence ranks among the highest resolutions achieved in any AGN in terms of Schwarzschild radii.

Acknowledgements. We thank R. W. Porcas for useful discussions throughout the project and for his careful review of the manuscript. His critique resulted in substantial improvements.

\section{References}

Alef, W. 1988, in The Impact of VLBI on Astrophysics and Geophysics, ed. M. J. Reid, \& J. M. Moran (Kluwer), IAU Symp., 129,523

Appl, S., \& Camenzind, M. 1993, A\&A, 270, 71

Blandford, R. D., \& Königl, A. 1979, ApJ, 232, 34

Bower, G. C., Falcke, H., Herrnstein, R. M., et al. 2004, Science, 304, 704

D'Addario, L. 2003, LAMA Memo Series, 802

Ferrarese, L., Ford, H. C., \& Jaffe, W. 1996, ApJ, 470, 444

Jones, D. L., \& Wehrle, A. E. 1997, ApJ, 484, 186

Jones, D. L., Wehrle, A. E., Meier, D. L., \& Piner, B. G. 2000, ApJ, 534,165

Jones, D. L., Wehrle, A. E., Piner, B. G., \& Meier, D. L. 2001, ApJ, 553,968

Junor, W., Biretta, J. A., \& Livio, M. 1999, Nature, 401, 891 
Kassim, N. E., Perley, R. A., Erickson, W. C., \& Dwarakanath, K. S. 1993, AJ, 106, 2218

Kellermann, K. I., Vermeulen, R. C., Zensus, J. A., \& Cohen, M. H. 1998, AJ, 115, 1295

Koide, S., Meier, D. L., Shibata, K., \& Kudoh, T. 2000, ApJ, 536, 668 Krichbaum, T. P., Alef, W., Witzel, A., et al. 1998, A\&A, 329, 873

Lobanov, A. P. 1998, A\&A, 330, 79

Lobanov, A. P., Krichbaum, T. P., Graham, D. A., et al. 2002, in Proc. of the 6th EVN Symposium, ed. E. Ros, R. W. Porcas, A. P. Lobanov, \& J. A. Zensus, 129

Ly, C., Walker, R. C., \& Wrobel, J. M. 2004, AJ, 127, 119

Marcaide, J. M., \& Shapiro, I. I. 1984, ApJ, 276, 56

Napier, P. J., Bagri, D. S., Clark, B. G., et al. 1994, Proc. IEEE, 82, 658

Narayan, R., Mahadevan, R., \& Quataert, E. 1998, in Theory of Black Hole Accretion Disks, 148
Porcas, R. W., \& Rioja, M. J. 2002, in 6th European VLBI Network Symposium on New Developments in VLBI Science and Technology, ed. E. Ros, R. W. Porcas, A. P. Lobanov, \& J. A. Zensus (Max-Planck-Institut für Radioastronomie), 65

Shapiro, I. I., Wittels, J. J., Counselman, C. C., et al. 1979, AJ, 84, 1459

Thompson, A. R., Moran, J. M., \& Swenson, G. W. 2001, Interferometry and synthesis in radio astronomy, 2nd ed. (New York: Wiley-Interscience), 692

Walker, R. C. 1995, in Very Long Baseline Interferometry and the VLBA, ed. J. A. Zensus, P. J. Diamond, \& P. J. Napier (ASP), ASP Conf. Ser., 82, 133

Yeh, K. C., \& Liu, C. H. 1982, Proc. IEEE, 70, 324 